\title{
Vibration Test of Airplane Moving Surface with Wireless Sensor
}

\author{
HE Jing, MA Yaping, LIU Ming, LI Tielin, ZENG Xingchang \\ Chinese Flight Test Establishment, Xi'an 710089, China \\ E-mail address: cfte_hj@126.com
}

\begin{abstract}
In flight test it is important to guarantee airplane flight safety through monitoring its structure vibration. But it is a challenge to monitor vibration for the airplane moving surface due to its large movement with composite materials and limited structure loading. It is difficult to use the conventional wire sensors and data acquisition system because of its large size and complicating wire routing. We design a test system based on micro- structure wireless sensor network with signal sensing, coding, storage and power supply to acquire data simultaneously through at several nodes with wireless time synchronization. The result of flight test show that the system is reliable with accurate data acquisition for vibration test of airplane moving surface.
\end{abstract}

Keywords: wireless network, vibration, time synchronization, MEMS, test technology

\section{Introduction}

The wireless network testing technology is considered as another hot spot in the field of industrial control after field bus and industrial Ethernet technology. As one of the most dynamic new technologies in the world, [1-3], its low cost, high integration, high flexibility and high reliability have solved the complicated connection, a lot of wiring, difficult installation and maintenance work for wired test. Therefore, all countries are competing for development in many application fields.
At present, the application of wireless intelligent testing technology is mainly focused on environmental monitoring and protection of medical care [4-5], large building structure [6-9] (such as Figure 1 bridge health monitoring) and military fields, and the application of state testing on aircraft structure is still applied less.

The health monitoring and structural state test of the aircraft is an important guarantee for the safety flight of the aircraft, and the wireless intelligent testing technology provides an effective method for the health monitoring and structural state testing of

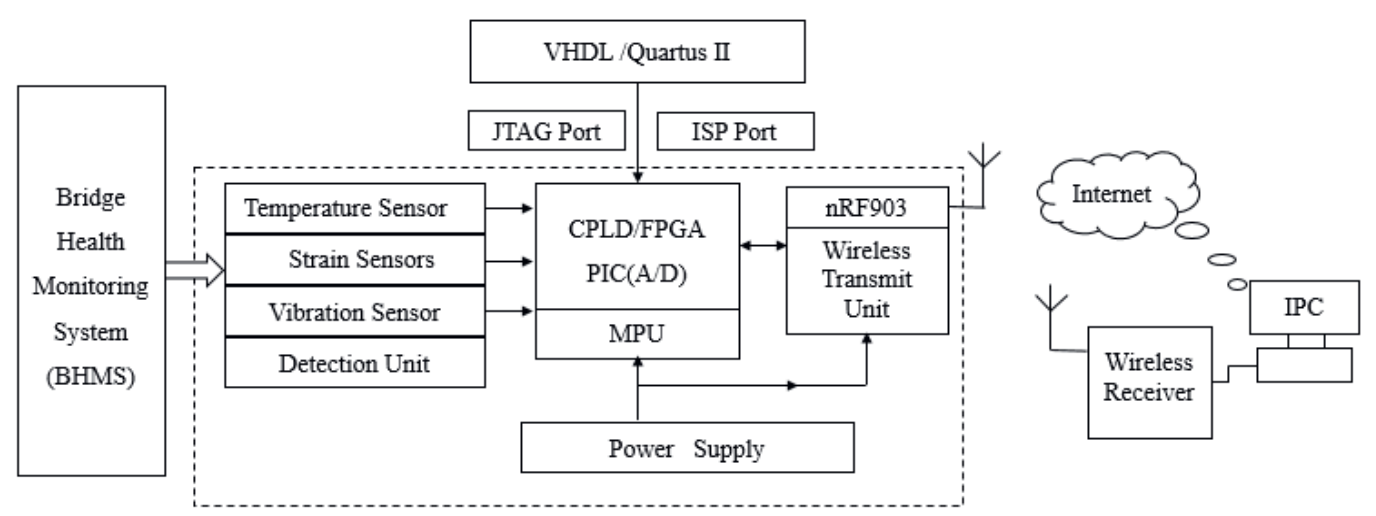

Fig. 1 Schematic diagram of wireless intelligent health monitoring for a large bridge 
the aircraft. The vibration test of aircraft is an important basis for studying the strength, deformation, mechanical fatigue and impact resistance of aircraft key components, which is important to the flight safety of an aircraft. Therefore, it is important to acquire the data of the vibration which is used for the study of the characteristics of vibration on the aircraft structure.

In this paper, the vibration characteristics of aircraft flap surface are monitored. Due to the continuous movement of the plane's flap wing during the flight, and the material of the flap is complex, the bearing capability of the structure is limited. The traditional cable sensor and data acquisition system have large volume and cumbersome wiring, which limits its application. Therefore, the wireless intelligent vibration testing technology is adopted by the vibration characterristics test of the flap surface.

\section{Vibration test with airborne wireless network}

2.1 The diagram and main technical specifications of airborne wireless vibration testing system

\subsubsection{Airborne wireless vibration testing system \\ The diagram of the airborne wireless vibration testing system is shown in Figure}

2. The system consists of wireless intelligent sensor nodes, wireless control unit and measurement and control terminal. The wireless intelligent acceleration sensor node sends the data to the wireless control unit through the $2.4 \mathrm{G}$ wireless communication, and the wireless control unit can connect 50 wireless intelligent sensor nodes at most. The wireless control unit transmits the data to the ground test computer through the Ethernet interface. Besides test wireless control unit can also be connected to telemetry system through RS422 interface in flight test.

\subsubsection{The main performance of the system}

- 50 remote wireless sensors connection at the same time;

- Each wireless sensor records data according to data sampling time sequence;

- The system can receive time from external IRIG-B code, and the synchronization accuracy is better than 20us;

- Wireless sensor integrates signal sensing, acquisition, recording and power supply;

- Continuous recording time: 15 hours;

- The measurement range and the sampling rate can be set up in software;

- Working status is displayed;

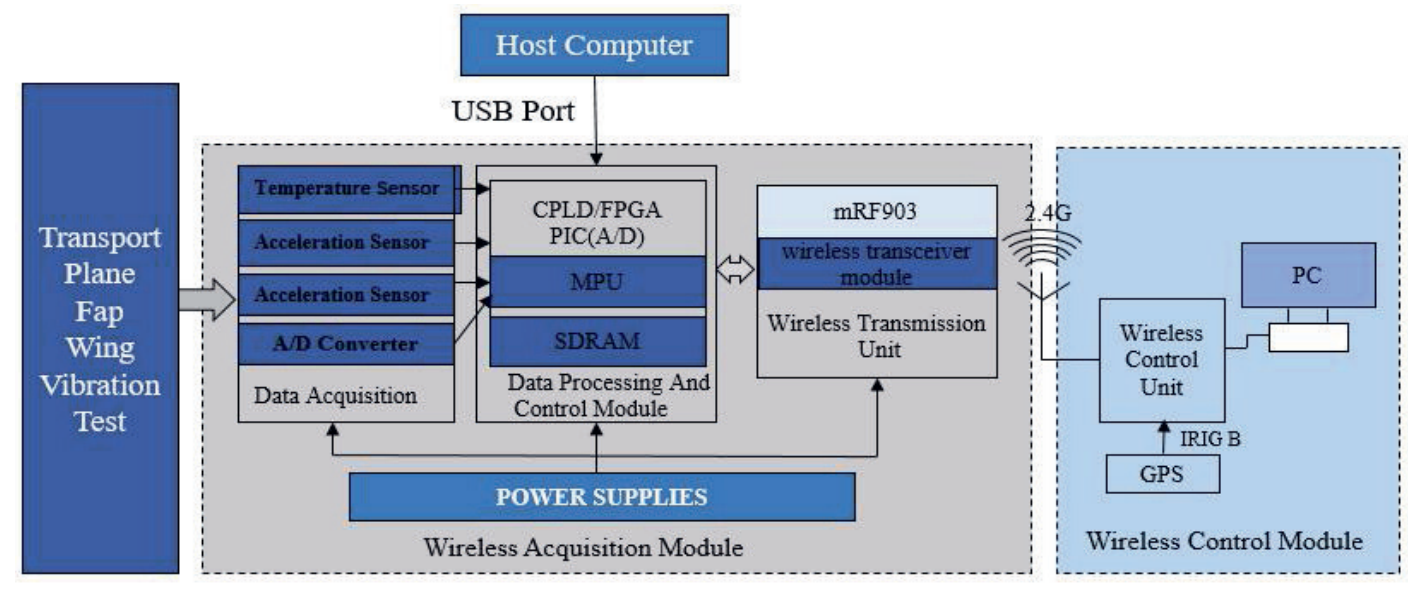

Fig. 2 Schematic diagram of airborne wireless intelligent vibration testing system 
- Data logging supports the different type format for data, TXT, CSV format. Software should have online help, whose content is comprehensive and convenient to search. The contents should include description of system function, software usage and software function.

\subsection{Wireless MEMS acceleration sensor}

The research of wireless intelligent vibration testing technology is studied in this paper. The three axis MEMS accelerometer is used as a micro sensor unit for signal acquisition, data processing and data storage. It follows the module design. The sensor is divided into data acquisition module, CPU module, power module and wireless transmission module, as shown in figure 3 .

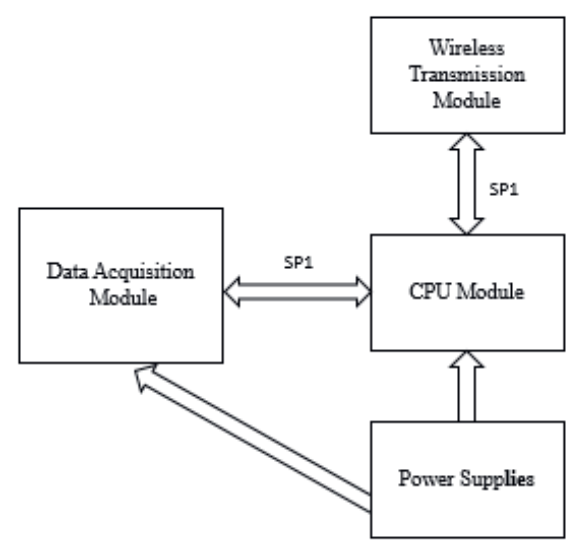

Figure 3 modular structure of wireless intelligence sensor

The sensitive device selects the MEMS acceleration chip, the signal conditioning part uses low power amplifier to build the two order of Butterworth low pass filter, and uses analog capacitor switching to realize the transformers of four different frequencies.

The CPU module is the core control part of wireless sensor, which completes data acquisition, data processing, data storage, power management, wireless communica- tion protocol and so on.

\subsection{Wireless control unit}

The wireless control unit is mainly composed of CPU, power module, Ethernet communication module, wireless transcei- ver module and synchronization module. The schematic diagram is shown in Figure 4.

The wireless control unit synchronize the nodes through the IRIG-B code and synchronize with the airborne test system time. The 28V DC power of the aircraft is transformed into $5 \mathrm{~V}$ and $3.3 \mathrm{~V}$ by means of DC-DC conversion and linear regulator.

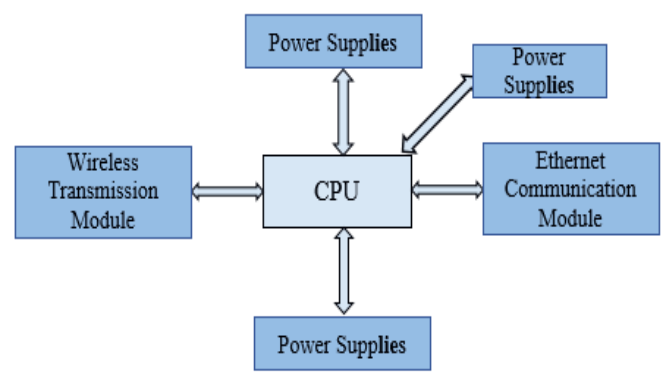

Figure 4. The schematic diagram of the wireless control unit

2.4 System time synchronization technology

In airborne wireless intelligent vibration testing system, time synchronization mechanism is a key technology, and each sensor node has its own local time. The sensor nodes use the crystal oscillator interrupt counter to get the local time. Because the frequency error and the initial timing time of the crystal oscillator are different, the local time between the nodes may be different, and the interference of the temperature and the electromagnetic wave etc. will also cause the time deviation between the nodes. The key to obtain the correct clock in time synchronization tech- nology is how to calculate the frequency deviation correctly and phase deviation between the local time or the physical time and local clock. Usually the frequency deviation and 
phase deviation are corrected by the transmission of synchronous information, but the time delay will be generated in the transmission of synchronization information. The uncertainty of the delay will affect the synchronization accuracy to a great extent. Therefore, calculating the delay of synchronization information correctly is the key step to improve the synchronization accuracy. The delay of synchronous information includes transmission delay, access delay, propagation delay and acceptance delay, as shown in Figure 3.

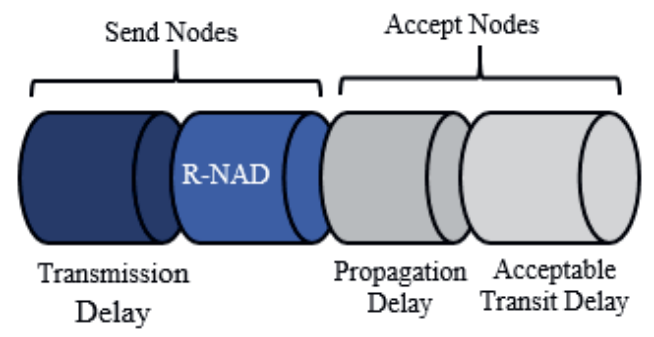

Figure3 Time delay in a wireless channel

In order to solve the problem of asynchronous data time stamp in the test system, this project proposes a solution, which uses the DMTS method to broadcast synchronous information packets with time delay measurement, as shown in Figure 4. A synchronous packet is sent every second through the wireless base station control synchronization accuracy can reach 20 microseconds.

\section{System software}

The wireless control unit is the control center of the wireless acquisition system. It operates the wireless protocol stack, controls all wireless sensor nodes, receives the synchronous clock from the airborne synchronization clock, sends the synchronization packets to the sensor nodes, receives all the data of the nodes, and runs the TCP protocol stack, and sends instruction and receives data through the Ethernet and the monitoring computer. The main functions are as follows:

a) The functions of search, node query, parameter setting, data collection and sensitivity configuration;

b) The functions of data storage query, data download, data erasure and so on;

c) Supports Win7/Win10 operating system, downloads data through USB

d) interface, and supports data format with data, TXT, CSV etc;

e) Data analysis software: time domain, frequency domain analysis, batch processing, wavelet transform and other functions.

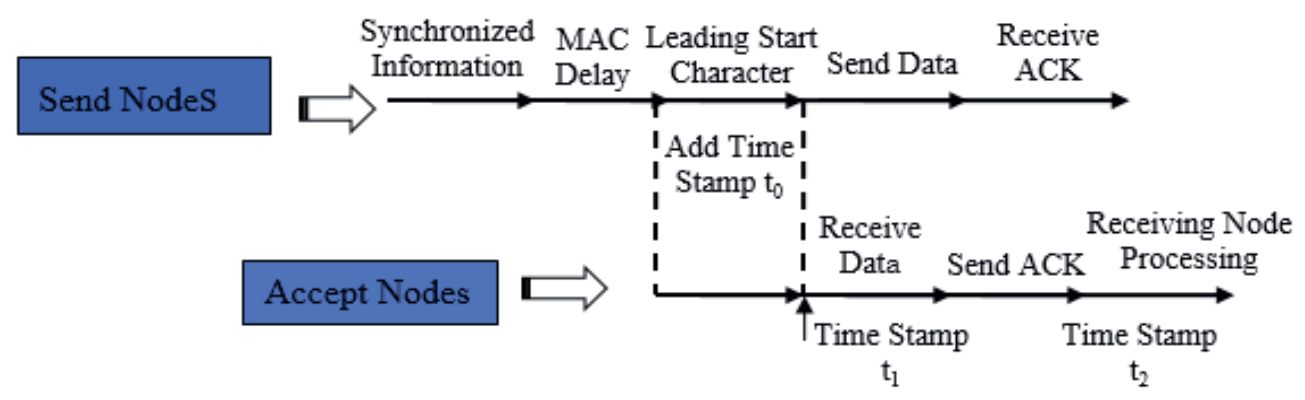

Figure 4 DMTS method broadcast synchronization packet delay measurement

unit, after the sensor node receives the synchronous packet, the initial value of the local timer is adjusted, thus the initial value of the local timer is adjusted. The time between each node is consistent, and the

\section{Test and Verification}

By the design of wireless network measurement node (wireless acceleration sensor), wireless control unit and 
measurement and control terminal software, the airborne wireless vibration testing system is constructed, and the time synchronization of wireless network nodes is studied by using the time delay measurement through synchronous packet broadcast based on DMTS method. The airborne wireless vibration test system can achieve $0 \sim 5 \mathrm{KHZ}$ sampling rate and $0 \sim$ $10 \mathrm{~g}$ vibration measurement. The wireless vibration network realizes the measurement of 18 wireless vibration sensor nodes at the same time. The data acquisition of each node is completely synchronized and has no time delay. The airborne wireless vibration testing system is verified on the aircraft to meet the test requirements of aircraft movement surface vibration characteristics.

\section{Conclusion}

In this paper, the MEMS micro sensor signal testing system based on wireless network is proposed from the point of view of system structure, hardware design, time synchronization and measurement and control software. The system is proved stable by the test of flight, reliable and high time synchronization precision, which solves the wiring problem in some cases of tests. It has a certain value to promotion.

\section{Reference}

[1]. Ren Zhi Ling, Zhang Guangquan, Lin Dong, et al. Overview of wireless sensor network applications [J]. sensors and Microsystems, 2018 (3).

[2]. Ren Fengyuan, Huang Haining, Lin Chuang, et al. Journal of [J]. Software of wireless sensor network, 2003, 14 (7): 1282-1291.

[3]. Potdar, Vidyasagar, Sharif, et al. Wireless Sensor Networks: A
Survey[J]. Computer Networks-The International Journal of Computer and Telecommunications Networking, 2009, 38(4):393-422.

[4]. Kung $\mathrm{H}$ Y, Hua J S, Chen C T. Drought Forecast Model and Framework Using Wireless Sensor Networks[J]., National Taiwan University biological resources and Agricultural Institute Experimental Forest Research Report, 2006, 20 (2):

[5]. Hande A, Polk T, Walker W, et al. Self-Powered Wireless Sensor Networks for Remote Patient Monitoring in Hospitals[J]. Sensors, 2006, 6(9):1102-1117.

[6]. Lee R G, Chen K C, Chiang S S, et al. A backup routing with wireless sensor network for bridge monitoring system[J]. Measurement, 2007, 40(1):55-63.

[7]. Yuan S, Lai $X$, Zhao $X$, et al. Distributed structural health monitoring system based on smart wireless sensor and multi-agent technology[J]. Smart Materials \& Structures, 2005, 15(1):1.

[8]. Chintalapudi K, Fu T, Paek J, et al. Monitoring civil structures with a wireless sensor network[J]. IEEE Internet Computing, 2006, 10(2):2634.

[9]. Ye Weisong, Yuan Shenfang. Application of wireless sensor network in structural health monitoring. Journal of [J]. Sensing Technology, 2006, 19 (3): 890-894. 